\title{
Equipment is cheap, but the field must develop and support common software for psychological research
}

\author{
WALTER SCHNEIDER \\ University of Pittsburgh, Pittsburgh, Pennsylvania
}

\begin{abstract}
Over the last 18 years, the commercial sector has provided psychology with very advanced equipment at low cost. It is unlikely, however, that the commercial sector will provide psychology with the special-purpose software that is needed for data acquisition. Software costs are now the greatest cost of data acquisition. Psychology needs common software used by many researchers. Efforts and funds must therefore be concentrated so that comprehensive software systems can be developed and supported. Software systems should provide open architectures to allow individual researchers to add specialized functions within an integrated environment.
\end{abstract}

In the 18 years since I joined the Society for Computers in Psychology, there have been great changes in computing. The hardware is now commonplace, but we need to find ways to develop and fund common software. My attendance at the meetings and my work in developing several software systems have provided me with some perspectives and hopes for the future.

Computer hardware has become a commodity of minor cost in equipping a laboratory. In 1977, the hardware on my first major grant took up about $75 \%$ of the direct-cost budget. In my last research grant, the hardware amounted to less than $5 \%$, and all of my data collection is currently done with computerized procedures. For my first grant, I spent weeks evaluating potential computers. Now I think about computers in about the same way as I do about buying a commodity like paper. Who has the best price this week, and who can deliver it in a case with the right size? In fact, I now spend more on photocopying costs than I do on purchasing computer equipment. Let me illustrate how insignificant the cost of raw computing power has become. I am developing a general response box interface that includes a microprocessor. The cost of the microprocessor chip (about \$2) is actually less than the cost of having the holes drilled in the printed circuit board $(40$ pins at $\$ 0.10 /$ hole) to mount the processor. Factors other than hardware costs now limit the use of computers.

Psychology benefits from the development aimed at commercial markets. The personal computer industry is a multibillion dollar industry that turns out tens of millions of computers annually. The commercial sector has invested billions that can be amortized over millions of

Inquiries regarding this paper should be sent to Walter Schneider, LRDC Building, 3939 O'Hara St., Pittsburgh, PA 15260.

Note: The author has a direct financial interest in one or more of the software products described in this paper.-Editor potential users. This provides us with very powerful general-purpose equipment at low cost.

The commercial sector will not meet our specialized needs. In the early 1970 s, vendors such as DEC were concerned about psychology. They sent salesmen to our early meetings. The last time that I remember a vendor present at the meeting was $1972 .{ }^{1}$ When the DEC representative was asked why only one DEC person was there that year, he said something like "All of psychology represents less sales than one good bank." If every member of the Psychonomic Society would buy a computer each year, it would represent less than $0.02 \%$ of the market. Ten years ago, it was easy to buy a vector-display scope to refresh the screen every millisecond. Now, I know of no vendor for IBM or Mac vector-display devices. It was easier to get millisecond timing out of older computers such as the PDP-8 than out of a modern-day Mac. In business environments, a .1-sec delay on keyboard entry is fast enough, but in psychology, we often want 0.001 -sec delays. But since psychology represents a trivial market, we have no clout in the commercial sector.

We have a de facto hardware standardization. Five years ago, there were many different machines. Today, the vast majority of psychological research computing is done on IBM PC compatibles and Macs. This sets the stage for common software standardization.

The biggest single cost of computerized research is software development. In an informal survey of some of my colleagues who conduct extensive computerized research, it is estimated that software development runs around $80 \%$ of the total data-acquisition cost in funds and time.

It is very difficult to cover the costs of extensive software development specialized for our field. To develop a complex system requires writing large amounts of code. For example, early versions of the MEL system, an experiment generator and analysis system (Schneider, 1988) involved over 100,000 lines of code. It is estimated that to write a 100,000 -line coding system would cost over 
$\$ 1,000,000$ at commercial rates (professional programmers have a labor rate of $\$ 30 / \mathrm{h}$, which includes benefits and indirect costs, and average 3 lines of debugged and documented code per hour). The cost of writing manuals exceeds $\$ 100 /$ page and a substantial manual (500 pp.) can cost $\$ 50,000$. To put this in perspective, the software development and documentation costs might amount to about $\$ 428$ ( $\$ 1.05$ million/2,400 researchers) per member of the Psychonomic Society. This cost is not ridiculous; it is about the cost of attending the annual meeting. If the software were specialized so that only one in five would purchase it, however, the cost would be $\$ 2,188$. I know of only one profitable software package (Mathematica) that covers its costs from primarily academic sales.

It is unreasonable to expect commercial firms to give us low-cost software as they once gave us low-cost hardware. It is up to our field to cover the cost of our specialized software needs.

Psychology has paid a heavy price for not developing common software usable by many researchers. In psychology, we have not had a common software environment. Think for a moment how less efficient computer science would be if every faculty member invented and used his or her own personal language. Algorithms could not be transferred. Young faculty would have to spend their first few years creating a specialized language. Students could not move from laboratory to laboratory, because each lab would use a different language. In psychology, it is almost this bad. Although we do not usually invent totally new languages, we do invent specialized subroutine packages that are unique to each laboratory.

When common software is not developed, there is little cumulative leaming of the field. It seems as if at nearly every meeting of the Society we have had several talks on implementing a "millisecond timer." It is very discouraging to hear a talk about something that we heard about years earlier. Even worse is to see people produce buggy procedures (e.g., not knowing how to read the IBM hardware clock without stopping it) that were in fact fixed years earlier.

We need less discussion of software algorithms and more supported distribution of solutions. It takes a lot longer to teach someone to program a millisecond timer than it does to link it. I do not worry about how the bus protocol in my computer is implemented; someone solved it, and I buy the solution. If a solution can be made available, do so and communicate its limits (e.g., timing accuracy). Spare other researchers the grief of going through the trouble you did to implement the solution. It is important that the people who provide the solution take on the responsibility of upgrading and supporting the solution (e.g., I once got code for a millisecond timer, but the author did not intend to update the code when a new generation of Macs became available that were incompatible with the earlier solution).

We need to pay more, not less, for specialized software. To illustrate, assume you could set the price for the software you buy. Assume there are 500 researchers who will purchase the system. Hence, your contribution will be multiplied 500-fold. If you set the price at $\$ 10$, you could buy 1 month of development effort; $\$ 100$ would buy 1 year of development; and $\$ 1,000$ would buy 10 years. Now, consider how hard it is for you to get $\$ 1,000$ out of your institution or granting agency relative to getting 5 years of a programmer's salary. ${ }^{2}$ What price would you set? By increasing the price of software, we cari concentrate development efforts so that the code need only be written, debugged, and documented once rather than hundreds of times, and so that researchers can spend more time on research and less on the implementation of software.

The field gains little from small-scale, isolated development efforts. The first development effort I engaged in (Schneider \& Scholz, 1973) involved a small system that I tried to give away; I got only one taker, who never used it. The field badly needs software development, and writing software that other colleagues could use represents a potentially significant contribution to the field. However, if you are unwilling to commit several person years to the project, it is unlikely to service even a few researchers. Operating in an open architecture is likely to be more effective (see below).

There are few large-scale research sofiware development efforts in the United States, and there should be more. It would be cost-effective for some government or foundation to fund the development of research support, rather than pay for many small-scale development efforts. There are government funds to support the development of instructional software and research, but generally not the development of software infrastructure for research. Other countries, such as the Netherlands and England, directly support institutes for the development of psychological software. There is one important source available in the United States for software development outside the university. The Small Business Research Initiative (SBRI) program provides initial (up to $\$ 50,000$ ), and occasionally follow-up (up to $\$ 500,000$ ), funding support for research software development. The PC Stereoscope group received one of these grants to develop software and hardware for visual psychophysics.

We need an open architecture and shared development efforts based in a small number of standardized sofiware environments. In order to develop more comprehensive research tools, we need to develop systems for research that solve most of the common problems and allow augmentation for special functions. Almost all computerized research requires precise timing, high-speed displays, data storage, and analysis. This is the core that can be developed to support a wide range of users. Many researchers have specialized needs such as eye-movement tracking, control of video tape recorders, network communications for multiple users, brain-wave monitoring, and speech input/output. Currently, researchers often spend many person years developing isolated systems used in single laboratories. If a standardized software environment existed, researchers would be able to write modules that could be linked to existing software, a process that would 
consume substantially less time. We need individuals who have the scientific expertise to develop specialized software (e.g., for speech synthesis) and develop linkable modules that many researchers can use on a common software foundation. The ability to link specialized routines has been added to integrated systems such as the MEL system and Apple-Psych (Osgood, 1988). Individual research grants can cover the cost of several person months to develop specific subroutines for research purposes. Later, these specific routines can be made available to other researchers using related paradigms. No individual or organization can develop all of the needed software. However, if we develop open standard software environments, we can have cumulative growth in our software tools.

In the future, experimental data-acquisition software should become as standardized as statistical software is today. Few researchers find it cost-effective to develop their own statistical software. They use established packages such as BMDP, SAS, and SPSS or spreadsheets such as Lotus 1-2-3. These have become standard "off the shelf solutions" for analysis procedures. I hope we will have a few experiment-generator packages that are commonly used by many researchers (see Butler, 1988, for a review). The MEL system, currently used at 700 sites, is perhaps the first of a set of standardized software packages for psychological data acquisition.

The future is bright, in that common hardware and software tools will empower psychologists to quickly and cheaply investigate a wide range of research questions. The hardware is becoming cheap and standardized. There are several initiatives to produce standardized software environments. As the field builds open modular research software, computerized research will be affordable and operable by nontechnical psychologists. The expenses for computerized psychological research are dropping. The hardware and software for a first-rate professional laboratory costs under $\$ 4,000$, approximately the cost of a teaching assistant for a semester. In the future, the software will be available to allow the researcher to be running complex experiments within the 1st month and analyzing the data within minutes of completion of the experiment. Researchers with small budgets will be able to investigate questions that could hardly have been dreamed of 5 years ago. They will also be able to contribute the cumulatively growing set of routines to enhance psychological data acquisition.

\section{REFERENCES}

BUTLER, D. L. (1988). A critical evaluation of software for experiment development in research and teaching. Behavior Research Methods, Instruments, \& Computers, 20, 218-220.

OsGOOD, G. (1988). Generalizing the Apple-Psych system. Behavior Research Methods, Instruments, \& Computers, 20, 155-157.

SCHNEIDER, W. (1988). Micro Experimental Laboratory: An integrated system for IBM PC compatibles. Behavior Research Methods, Instruments, \& Computers, 20, 206-217.

SChNeIder, W., \& Scholz, K. W. (1973). Requirements for minicomputer operating systems for human experimentation and an implementation on a 4K PDP-8 computer. Behavior Research Methods \& Instrumentation, 5, 173-177.

\section{NOTES}

1. Vendors attended and exhibited at the meetings of the Society for Computers in Psychology until the early 1980s.

2. For the purpose of calculation, I assume that a typical researcher would use less than half of the features developed for a system designed to meet the needs of 500 researchers. 